\title{
Free expansion of Bose-Einstein Condensates with a Multi-charged Vortex
}

\author{
R. P. Teles, F. E. A. dos Santos, M. A. Caracanhas, and V. S. Bagnato \\ Instituto de Fï̈œica de Sï̋œ Carlos, USP, Caixa Postal 369, \\ 13560-970 Sį̈œо Carlos, Sį̈œо Paulo, Brazil
}

\begin{abstract}
In this work, we analyze the free expansion of Bose-Einstein condensates containing multi-charged vortices. The atomic cloud is initially confined in a three-dimensional asymmetric harmonic trap. We apply both approximate variational solutions and numerical simulations of the Gross-Pitaevskii equation. The data obtained provide a way to establish the presence as well as the multiplicity of vortices based only on the properties of the expanded cloud which can be obtained via timeof-flight measurements. In addition, several features like the evolution of the vortex core size and the asymptotic velocity during free expansion were studied considering the atomic cloud as being released from different harmonic trap configurations.
\end{abstract}




\section{INTRODUCTION}

The extension of quantum phenomena into macroscopic scales is responsible for a whole class of effects such as superconductivity, superfluidity, and Bose-Einstein Condensation, which played central roles in the last-century physics. The production of the first BoseEinstein condensates (BECs), using rubidium [1] and sodium [2] atoms, turned possible the realization of experiments involving macroscopic quantum phenomena with unprecedented level of control of the external parameters.

Vortices in BECs are topological defects characterized by a quantized angular momentum. A conventional method for generation of such defects consists in confining the condensed atomic cloud into a rotating trap. It turns out that, for angular velocities higher than a critical value $\Omega_{c}$, vortex states become energetically favorable, thus inducing the creation of quantized vortices [3 6]. Experimental realizations of condensed alkali atoms confined by more general time-dependent potentials allowed the observation not only of vortex lattices but also of quantum turbulence [2, 7-10]. Since quantum turbulence is characterized by the presence of a self-interacting tangle of quantized vortices, the correct understanding of dynamics, formation, and stability of vortices have shown to be of paramount importance [9, 11 14] being the subject of many theoretical works [10, 15 19]. In particular, the role of acoustic excitations generated by decaying multi-charged vortices in the development of turbulence is still an open question [20]. This work aims to provide a set of tools that helps to identify the presence as well as the charge of vortices in both turbulent and non-turbulent clouds observed using time-of-flight pictures.

The radius of vortex cores are typically of the order of the healing length of the condensate. Such a small size makes in situ observations very difficult. The most common method for visualization of vortices in BECs relies on the so called time-flight pictures which can be obtained after releasing the condensed cloud from its trap and letting it expand freely for some time, typically tens of milliseconds [8, 17, 21 23]. To determine the charge multiplicity of vortices in confined clouds using time-of-flight pictures, it is necessary to establish the correct connection between the features of the trapped and expanded clouds.

Charged vortices have regions of stability which depend on some of the sample characteristics such as: winding number, trap anisotropy and intensity of the atomic interaction[24-29]. The majority of the theories of multi-charged vortex stability do not take into account the 
presence of thermal cloud. In realistic experiments, the coupling between condensate and thermal cloud is an important key to stability. In general, dissipation makes multi-charged vortices split into singly charged vortices[27, 30]. However, some research groups have implemented techniques to overcome this issue, as for example: implementing a tightly focused resonant laser[31], using a blue-detuned laser beam which compensates the gravity[32], and by the application of a Gaussian potential peak along the vortex core [33].

In this paper, we considered the expansion of a BEC containing a multiply charged vortex at its center. The main calculations are performed by using a variational method which takes into account the presence of non-fundamental vortices. A similar work for single charged vortices in two-dimensional condensates was done by Lundh et al. [34] and a more numerical approach was employed by Dalfovo et al. in Ref. [35].

This work is divided as follows: section I is this introduction. Section II presents the variational method used in this work. In the section III, we discuss the dynamical equations for the expanding condensate. Finally, in section IV there is a general discussion of our results.

\section{VARIATIONAL METHOD}

At zero temperature, a Bose gas with scattering length $a_{s}$ much smaller than the average interparticle distance can be described by the Gross-Pitaevskii equation (GPE)

$$
i \hbar \frac{\partial \Psi(\mathbf{r}, t)}{\partial t}=\left[-\frac{\hbar^{2}}{2 m} \nabla^{2}+V(\mathbf{r})+U_{0}|\Psi(\mathbf{r}, t)|^{2}\right] \Psi(\mathbf{r}, t),
$$

with the harmonic trap in cylindrical coordinates given by $V(\mathbf{r})=\frac{1}{2} m \omega_{\rho}^{2}\left(\rho^{2}+\lambda^{2} z^{2}\right)$, where $m$ is the mass of the particles, $\lambda$ is an anisotropy parameter, and the coupling constant is $U_{0}=4 \pi \hbar^{2} a_{s} / m$. Following the variational principle, the Lagrangian density $\mathcal{L}$ which recovers the GPE for a complex field $\Psi(\mathbf{r}, t)$ can be written as

$$
\begin{gathered}
\mathcal{L}=\frac{i \hbar}{2}\left(\Psi^{*}(\mathbf{r}, t) \frac{\partial \Psi(\mathbf{r}, t)}{\partial t}-\Psi(\mathbf{r}, t) \frac{\partial \Psi^{*}(\mathbf{r}, t)}{\partial t}\right) \\
-\frac{\hbar^{2}}{2 m}|\nabla \Psi(\mathbf{r}, t)|^{2}-V(\mathbf{r})|\Psi(\mathbf{r}, t)|^{2}-\frac{U_{0}}{2}|\Psi(\mathbf{r}, t)|^{4} .
\end{gathered}
$$

In the variational method, the wave function $\Psi(\mathbf{r}, t)$ of a condensate containing one central vortex with charge $\ell$ is approximated by a trial function $\Psi_{\ell}(\mathbf{r}, t)$ which depends on a 
set of variational parameters $q_{i}=q_{i}(t)$ [36, 37]. This function can then be substituted into Lagrange function

$$
L=\int \mathcal{L} d^{3} \mathbf{r}
$$

This way, the time evolution of the parameters $q_{i}$ follows the Euler-Lagrange equations

$$
\frac{\partial}{\partial t}\left(\frac{\partial L}{\partial \dot{q}_{i}}\right)-\frac{\partial L}{\partial q_{i}}=0
$$

Here, we generalize the Gaussian trial function in Ref. [34] to the case of three-dimensional BECs with multiply charged vortices

$$
\Psi_{\ell}(\rho, \phi, z, t)=\left[\frac{N}{\pi^{\frac{3}{2}} \ell ! R_{\rho}(t)^{2 \ell+2} R_{z}(t)}\right]^{\frac{1}{2}} \rho^{\ell} e^{i \ell \phi} \psi_{G}(\rho, z, t) e^{i B_{\rho}(t) \frac{\rho^{2}}{2}+i B_{z}(t) \frac{z^{2}}{2}},
$$

with the function $\psi_{G}$ being given by

$$
\psi_{G}(\rho, z, t)=\exp \left(-\frac{\rho^{2}}{2 R_{\rho}(t)^{2}}\right) \exp \left(-\frac{z^{2}}{2 R_{z}(t)^{2}}\right) .
$$

If we consider $\ell=0$, we recover the vortex-free approximation proposed by Pï¿œrezGarcï¿œa et al. in Ref. [36]. In our case, however, the parameter $R_{\rho}$ is no longer the mean square root of $\rho$. Instead, it is related to $\sqrt{\left\langle\rho^{2}\right\rangle}$ according to

$$
\sqrt{\left\langle\rho^{2}\right\rangle}=\sqrt{\ell+1} R_{\rho}
$$

Here we define the vortex core $\xi$ as the healing length calculated at the center of the condensate without the central vortex. This assumption leads us to

$$
\xi=\ell \hbar \pi^{\frac{3}{4}} R_{\rho} \sqrt{\frac{R_{z}}{2 m N U_{0}}} .
$$

\section{DYNAMICAL EQUATIONS}

By substituting (5) into Eqs. (2) and (3), and then performing the spacial integrations, we obtain the Lagrange function for the variational parameters

$L=-N \hbar \omega_{\rho}\left\{\frac{(\ell+1)}{2}\left[\frac{1}{r_{\rho}^{2}}+\left(\dot{\beta}_{\rho}+\beta_{\rho}^{2}+1\right) r_{\rho}^{2}\right]+\frac{1}{4}\left[\frac{1}{r_{z}^{2}}+\left(\dot{\beta}_{z}+\beta_{z}^{2}+\lambda^{2}\right) r_{z}^{2}\right]+\frac{\gamma(2 \ell) !}{2^{2 \ell} \sqrt{2 \pi}(\ell !)^{2} r_{\rho}^{2} r_{z}}\right\}$ 
where the parameters were rescaled according to $R_{\rho}(t)=a_{o s c} r_{\rho}(t), R_{z}(t)=a_{o s c} r_{z}(t), B_{\rho}(t)=$ $a_{\text {osc }}^{-2} \beta_{\rho}(t)$, and $B_{z}(t)=a_{\text {osc }}^{-2} \beta_{z}(t)$. The harmonic oscillator length was defined as $a_{o s c}=$ $\sqrt{\hbar / m \omega_{\rho}}$, whereas the dimensionless iteration was defined according to $\gamma=N a_{s} / a_{o s c}$. The Euler-Lagrange equations (41) applied to the Langrangean (9) finally give us the equations of motion

$$
\begin{aligned}
\left(\dot{\beta}_{\rho}+\beta_{\rho}^{2}+1\right) r_{\rho} & =\frac{1}{r_{\rho}^{3}}+\frac{\gamma(2 \ell) !}{2^{2 \ell-1} \sqrt{2 \pi}(\ell+1) ! \ell ! r_{\rho}^{3} r_{z}}, \\
\beta_{\rho} & =\frac{\dot{r_{\rho}}}{r_{\rho}}, \\
\left(\dot{\beta}_{z}+\beta_{z}^{2}+\lambda^{2}\right) r_{z} & =\frac{1}{r_{z}^{3}}+\frac{\gamma(2 \ell) !}{2^{2 \ell-1} \sqrt{2 \pi}(\ell !)^{2} r_{\rho}^{2} r_{z}^{2}}, \\
\beta_{z} & =\frac{\dot{r_{z}}}{r_{z}} .
\end{aligned}
$$

By taking the time-derivative of Eqs. (11) and (13), the parameters $\beta_{\rho}$ and $\beta_{z}$ can be eliminated in such a way that these four equations can be reduced to the following two:

$$
\begin{aligned}
\ddot{r}_{\rho}+r_{\rho} & =\frac{1}{r_{\rho}^{3}}+\frac{\gamma(2 \ell) !}{2^{2 \ell-1} \sqrt{2 \pi}(\ell+1) ! \ell ! r_{\rho}^{3} r_{z}}, \\
\ddot{r}_{z}+\lambda^{2} r_{z} & =\frac{1}{r_{z}^{3}}+\frac{\gamma(2 \ell) !}{2^{2 \ell-1} \sqrt{2 \pi}(\ell !)^{2} r_{\rho}^{2} r_{z}^{2}} .
\end{aligned}
$$

\section{A. Free expansion}

By considering the stationary solution for the Eqs. of motion (14) and (15), we obtain the algebraic equations

$$
\begin{aligned}
r_{\rho 0}^{4} & =1+\frac{\gamma(2 \ell) !}{2^{2 \ell-1} \sqrt{2 \pi}(\ell+1) ! \ell ! r_{z 0}} \\
\lambda^{2} r_{z 0}^{4} & =1+\frac{\gamma(2 \ell) ! r_{z 0}}{2^{2 \ell-1} \sqrt{2 \pi}(\ell !)^{2} r_{\rho 0}^{2}} .
\end{aligned}
$$

The free expansion equations are obtained when the trap terms in Eqs. (14) and (15) are neglected, and thus they become

$$
\begin{aligned}
& \ddot{r}_{\rho}=\frac{1}{r_{\rho}^{3}}+\frac{(2 \ell) ! \gamma}{2^{2 \ell-1}(2 \pi)^{\frac{1}{2}}(\ell+1) ! \ell ! r_{\rho}^{3} r_{z}} \\
& \ddot{r}_{z}=\frac{1}{r_{z}^{3}}+\frac{(2 \ell) ! \gamma}{2^{2 \ell-1}(2 \pi)^{\frac{1}{2}}(\ell !)^{2} r_{\rho}^{2} r_{z}^{2}}
\end{aligned}
$$


The first and second terms in the r.h.s of (18) and (19) come from the kinetic and interaction terms in (2), respectively. From (18) and (19), we can also observe that the interaction term is dominant in the long-time limit, while the kinetic terms plays a role only at the first milliseconds of the expansion. This is however not the case when we consider extremely large values of $\ell$. In this case, the interaction terms can be neglected thus leading to equations identical to the ones describing the free expansion of an ideal gas

$$
\begin{aligned}
& \ddot{r}_{\rho}=\frac{1}{r_{\rho}^{3}}, \\
& \ddot{r}_{z}=\frac{1}{r_{z}^{3}},
\end{aligned}
$$

which have the simple solution

$$
r_{j}(\tau)=\sqrt{r_{j}(0)^{2}+r_{j}(0)^{-2} \tau^{2}}
$$

with $j=\rho, z$.

In order to check the accuracy of our results, we performed direct simulation of the GP equation using the Fourier spectral method in space where the Fourier components of $\Psi(\mathbf{r}, t)$ where computed using fast Fourier transformations[38]. In figure 1, our variational method is compared with the high-precision numerical simulation for expanding spherical as well as prolate condensates.

\section{RESULTS AND DISCUSSIONS}

The initial condition for the variational parameters where calculated from Eqs. (16) and (17) considering the radial frequency $\omega_{\rho}=2 \pi \times 207 \mathrm{~Hz}$. The time evolution of the parameters was obtained by numerically solving Eqs. (18) and (19) using the fourth-order adaptive Runge-Kutta method, with expansion times of the order of $30 \mathrm{~ms}$. These values where chosen in order to be consistent with our experiments using ${ }^{87} \mathrm{Rb} 87$ atoms [39].

Figure 2 shows the initial energy of the system for a fixed interaction parameter as function of the trap anisotropy measured by the parameter $\lambda$. It shows the monotonic growth of the condensate energy with the circulation of the vortex due to the extra kinetic energy and the larger volume occupied by the condensate with increasing $\ell$.

The vortex core evolution was analyzed considering its size to be given by Eq. (8). A disadvantage of this method comes from the fact that the static constraint (8) between the 


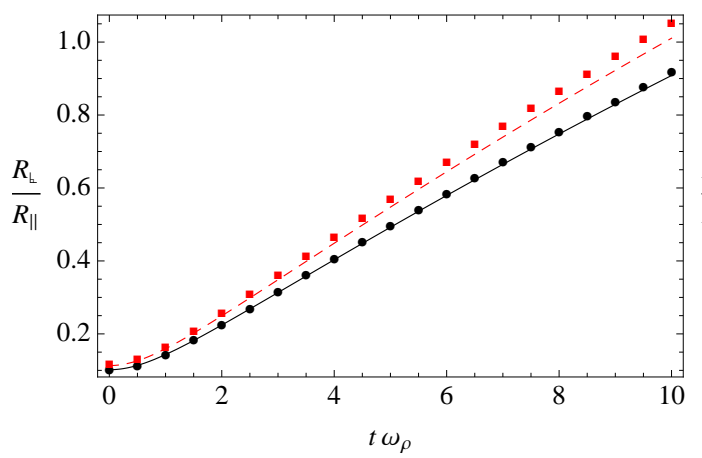

(a) Prolate shape $(\lambda=0.1)$.

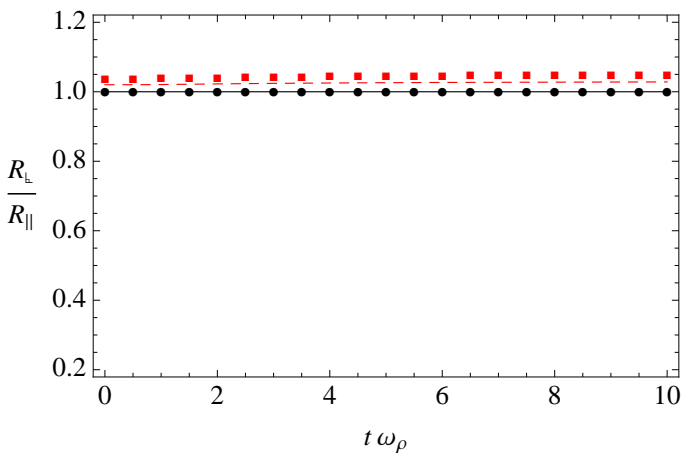

(b) Spherical shape $(\lambda=1)$.

Figure 1: (Color online) Comparison of the free expansion for $\gamma=800$ using the variational method and the the direct numerical simulation of GPE. The lines and marks come from the variational method and the numerical simulation of GPE, respectively. Full line and circle mark (black color) correspond to $\ell=0$, and the dashed line and square mark (red color) correspond to $\ell=2$.

vortex core and the cloud dimensions is considered to be also valid during the entire expansion time. At least in principle, our Ansatz can be improved by introducing an additional parameter which characterizes the core expansion independently. In practice, the introduction of such an additional parameter affecting the density profile of the cloud is a not a trivial task. Indeed, the phase of the condensate wave function must also be modified in order to reproduce superfluid current corresponding to the time-variant core size. However, since in this work our attention is restricted to static configurations and the expansion of the cloud, the approach used here work turns out to be appropriate as we can see from Fig. 1 .

In Fig. 3(a)-(c), the time evolution of the aspect ratio for different trap configurations and vortex circulations is depicted. Figure 3(c) shows how the growth of the dimensions of the cloud during the expansion are affected by the circulation of the central vortex. The higher the circulation of these vortices, the greater is the anisotropy introduced in the cloud shape. This effect is further increased during the cloud expansion.

In prolate condensates, Fig. 3a shows that the circulation has the effect of decreasing the time for the aspect ratio inversion due to the larger velocity field in the plane perpendicular to the vortex line before the expansion. In the opposite case, where the initial geometry is 


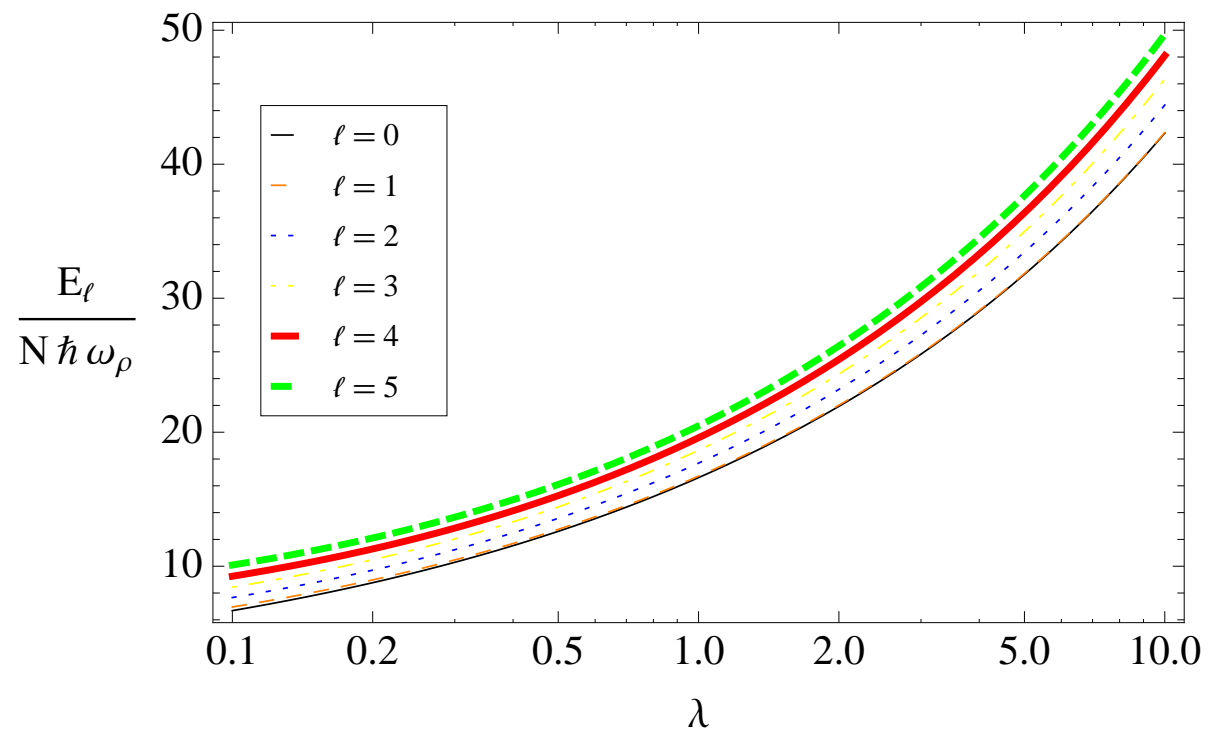

Figure 2: (Color online) Energy plotted as function of the trap anisotropy for different vortex circulations. The dimensionless interaction parameter is taken as $\gamma=800$. This corresponds to a condensate with $10^{5}$ atoms and s-wave scattering length $a_{s}=100 a_{0}$.

oblate, as in Fig. 3b, the circulation increases the time required for aspect ratio inversion.

In this work, the expansion of the vortex core is analyzed by evolving both the radial and axial radius using Eqs. (18) and (19), and then calculating the radius $\xi$ of the vortex according to (8). By taking the asymptotic solutions of Eqs. (18) and (19), it was also possible to extract the asymptotic expansion velocities along the radial and axial directions, as shown in Fig. 4. These information supply a method for determining the presence as well as the circulation of central vortices in an asymmetric cloud by simply looking at the asymptotic expansion velocities, which would require the repetition of the experiment considering different expansion time. An alternative method relies on dependence of the expansion dynamics on the circulation $\ell$ as depicted in Fig. 3d. In fact, Figs. 3(a)-(c) give a one to one correspondence between the aspect ratio of the expanded cloud and the multiplicity of the central vortex. This way, the multiplicity of the central core can be determined from aspect ratio obtained from time-of-flight pictures. 


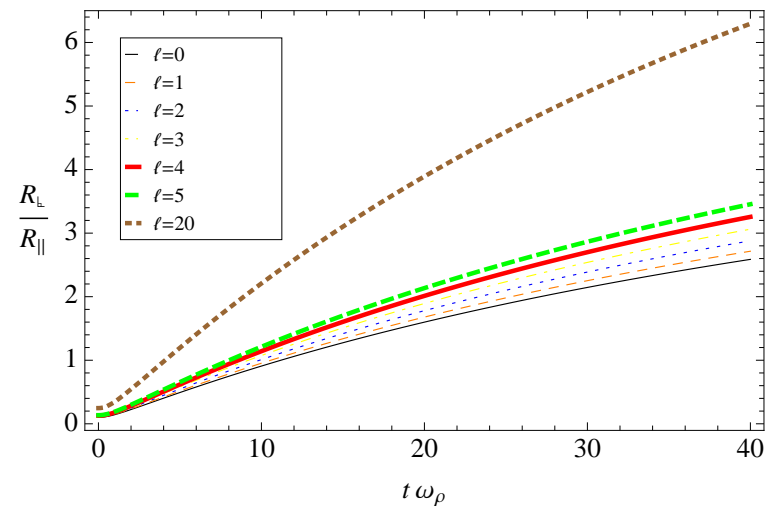

(a) $\lambda=0.1$

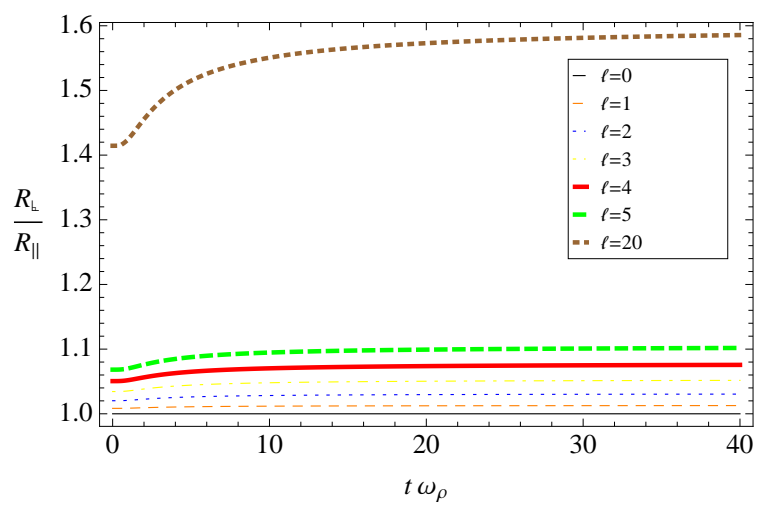

(c) $\lambda=1$

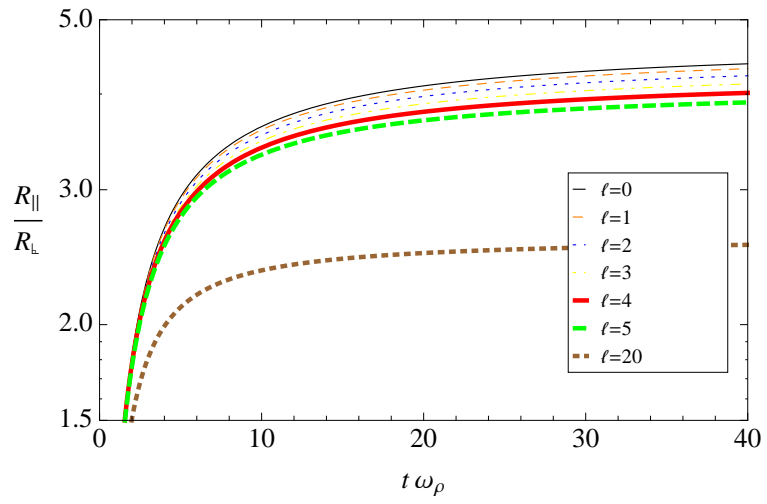

(b) $\lambda=10$

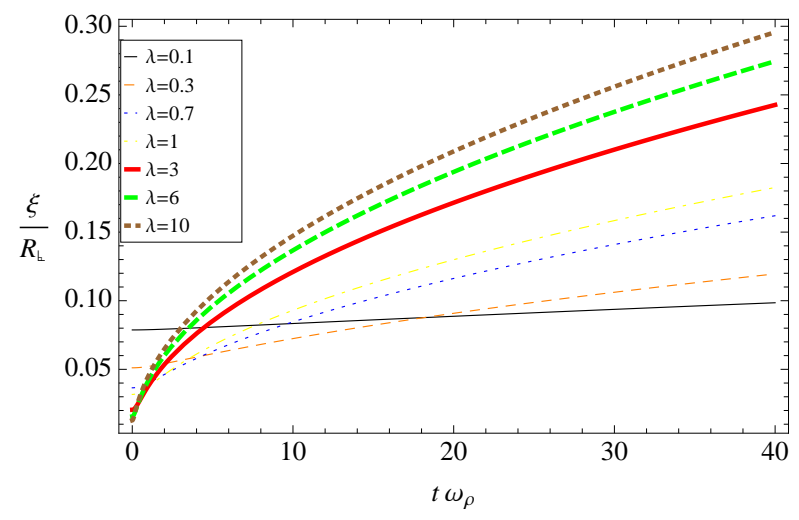

(d) $\ell=1$

Figure 3: (Color online) Aspect ratio evolution during free expansion for the respective trapped shapes: (a) prolate, (b) oblate and (c) spherical. They were calculated for $\gamma=800$. Frame $(\mathrm{d})$ represents the ratio between the vortex core and the radial radius during the expansion for several kind of trap shapes.

\section{Acknowledgments}

We acknowledge the financial support of from the National Council for the Improvement of Higher Education (CAPES) and from the State of São Paulo Foundation for Research Support (FAPESP). 

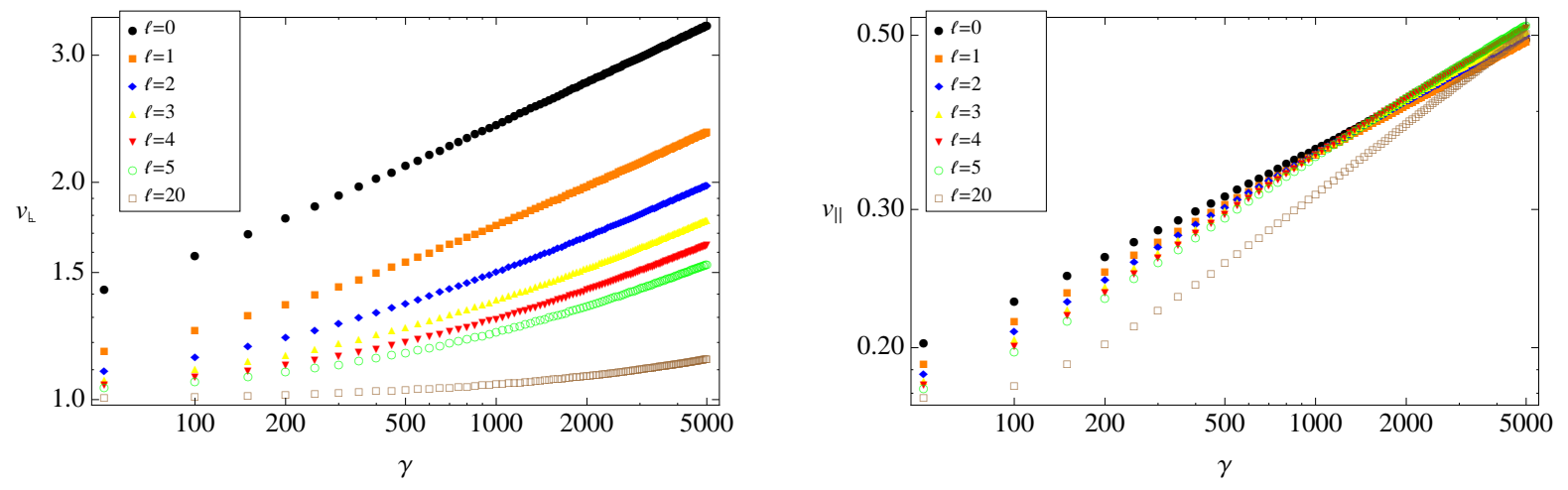

(a) $\lambda=0.1$
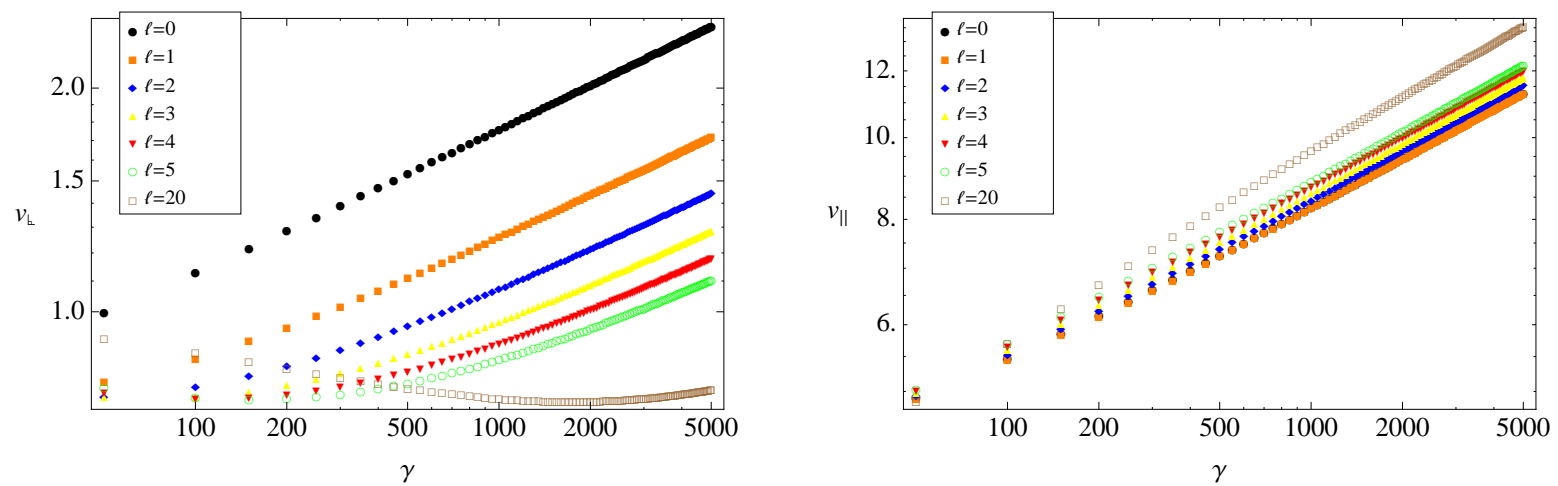

(b) $\lambda=10$

Figure 4: (Color online) The asymptotic behavior of expansion velocities as a function of $\gamma$ for initially prolate (a) and oblate (b) shapes.

[1] M. H. Anderson, J. R. Ensher, M. R. Matthews, C. E. Wieman, and E. A. Cornell, Science 269, 198 (1995).

[2] K. B. Davis, M. O. Mewes, M. R. Andrews, N. J. van Druten, D. S. Durfee, D. M. Kurn, and W. Ketterle, Physical Review Letters 75, 3969 (1995).

[3] P. Rosenbusch, D. S. Petrov, S. Sinha, F. Chevy, Y. C. V. Bretin, G. Shlyapnikov, and J. Dalibard, Physical Review Letters 88, 250403 (2002).

[4] K. W. Madison, F. Chevy, W. Wohlleben, and J. Dalibard, Physical Review Letters 84, 806 (2000).

[5] E. Hodby, G. Hechenblaikner, S. A. Hopkins, O. M. Maragò, and C. J. Foot, Physical Review 
Letters 88, 010405 (2001).

[6] S. Dtringari, Physical Review Letters 82, 4371 (1999).

[7] J. A. Seman, E. A. L. Henn, M. Haque, R. F. Shiozaki, E. R. F. Ramos, M. Caracanhas, P. Castilho, C. C. Branco, P. E. S. Tavares, F. J. Poveda-Cuevas, et al., Physical Review A 82, $033616(2010)$.

[8] P. W. Courteille, V. S. Bagnato, and V. I. Yukalov, Laser Physics 11, 659 (2001).

[9] E. A. L. Henn, J. A. Seman, G. Roati, K. M. F. Magalhães, and V. S. Bagnato, Physical Review Letters 103, 045301 (2009).

[10] C. J. Pethick and H. Smith, Bose-einstein condensation in dilute gases (Cambridge University Press, Cambridge, 2008), 2nd ed.

[11] C. F. Barenghi and Y. A. Sergeev, Vortices and turbulence at very low temperatures (SpringerWienNewYork, New York, 2008).

[12] R. Carretero-González, B. P. Anderson, P. G. Kevrekidis, D. J. Frantzeskakis, and C. N. Weiler, Physical Review A 77, 033625 (2008).

[13] D. H. J. O’Dell and C. Eberlein, Physical Review A 75, 013604 (2007).

[14] A. A. Svidzinsky and A. L. Fetter, Physical Review Letters 84, 5919 (2000).

[15] A. Aftalion, Vortices in bose-einstein condensates (Birkhäuser, Boston, Basel, Berlin, 2006. (Progress in Nonlinear Differential Equations and Their Applications, v. 67 )).

[16] J. R. Abo-Shaeer, C. Raman, and W. Ketterle, Physical Review Letters 88, 070409 (2002).

[17] E. A. L. Henn, J. A. Seman, E. R. F. Ramos, M. Caracanhas, P. Castilho, E. P. Olímpio, G. Roati, D. V. Magalhães, K. M. F. Magalhães, and V. S. Bagnato, Physical Review A 79, 043618 (2009).

[18] A. L. Fetter and A. A. Svidzinsky, Journal of Physics: Condensate Matter 13, R135 (2001).

[19] P. Rosenbusch, V. Bretin, and J. Dalibard, Physical Review Letters 89, 200403 (2002).

[20] I. Aranson and V. Steinberg, Physical Review B 53, 75 (1996).

[21] B. P. Anderson and P. C. Haljan, Physical Review Letters 85, 2857 (2000).

[22] F. Chevy, K. W. Madison, and J. Dalibard, Physical Review Letters 85, 2223 (2002).

[23] W. Ketterle, MIT Physics Annual pp. 44-49 (2001).

[24] H. Pu, C. K. Law, J. H. Eberly, and N. P. Bigelow, Physical Review A 59, 1533 (1999).

[25] D. L. Feder, C. W. Clark, and B. I. Schneider, Physical Review Letters 82, 4956 (1999).

[26] M. Möttönen, T. Mizushima, T. Isoshima, M. M. Salomaa, and K. Machida, Physical Review 
A 68, 023611 (2003).

[27] Y. Kawaguchi and T. Ohmi, Physical Review A 70, 043610 (2004).

[28] C. Josserand, Chaos: An Interdisciplinary Journal of Nonlinear Science 14, 875 (2004).

[29] P. Kuopanportti, J. A. M. Huhtamäki, V. Pietilä, and M. Möttönen, Physical Review A 81, $023603(2010)$.

[30] T. Karpiuk, M. Brewsczyk, M. Gajda, and K. Rzążewski, Journal of Physics B: Aomic, Molecular and Optical Physics 42, 095301 (2009).

[31] P. Engels, I. Coddington, P. C. Haljan, V. Schweikhard, and E. A. Cornell, Physical Review Letters 90, 170405 (2003).

[32] M. Kumakura, T. Hirotani, M. Okano, Y. Takahashi, and T. Yakuzaki, Physical Review A 73, 063605 (2006).

[33] P. Kuopanportti and M. Möttönen, Journal of Low Temperature Physics 161 (2010).

[34] E. Lundh, C. J. Pethick, and H. Smith, Physical Review A 58, 4816 (1998).

[35] F. Dalfovo and M. Modugno, Physical Review A 61, 023605 (2000).

[36] V. M. Pérez-García, H. Michinel, J. I. Cirac, M. Lewenstein, and P. Zoller, Physical Review A 56, 1424 (1997).

[37] V. M. Pérez-García, H. Michinel, J. I. Cirac, M. Lewenstein, and P. Zoller, Physical Review Letters 77, 5320 (1996).

[38] G. R. Dennis, J. J. Hope, and M. T. Johnsson, Computer Physics Communications 184, 201 (2013).

[39] E. A. de Lima Henn, Ph.D. thesis, Intituto de Física de São Carlos, Universidade de São Paulo, São Carlos (2008). 